\title{
Front Matter: Volume 10025
}

, "Front Matter: Volume 10025," Proc. SPIE 10025, Advanced Sensor Systems and Applications VII, 1002501 (9 January 2017); doi:

10.1117/12.2265123

SPIE. Event: SPIE/COS Photonics Asia, 2016, Beijing, China 


\title{
Advanced Sensor Systems and Applications VII
}

\author{
Tiegen Liu \\ Shibin Jiang \\ Rene Landgraf \\ Editors
}

\section{2-14 October 2016 \\ Beijing, China}

Sponsored by

SPIE

COS-Chinese Optical Society

Cooperating Organizations

Tsinghua University (China) - Peking University (China) • University of Science and Technology of China (China) Zhejiang University (China) • Tianjin University (China) • Beijing Institute of Technology (China) • Beijing University of Posts and Telecommunications (China) - Nankai University (China) - Changchun University of Science and Technology (China) • University of Shanghai for Science and Technology (China) - Capital Normal University (China) Huazhong University of Science and Technology (China) - Beijing Jiaotong University (China) • Shanghai Institute of Optics and Fine Mechanics (China) - Changchun Institute of Optics and Fine Mechanics (China) - Institute of Semiconductors (China) • Institute of Optics and Electronics (China) • Institute of Physics (China) • Shanghai Institute of Technical Physics (China) - China Instrument and Control Society (China) - Optoelectronics Technology Committee, COS (China) • SPIE National Committee in China (China) • Optical Society of Japan (Japan) • Optical Society of Korea (Korea, Republic of) - The Australian Optical Society (Australia) - Optics and Photonics Society of Singapore (Singapore) • European Optical Society

Supporting Organizations

CAST_China Association for Science and Technology (China)

NSFC-National Nature Science Foundation (China)

Published by

SPIE

Volume 10025 
The papers in this volume were part of the technical conference cited on the cover and title page. Papers were selected and subject to review by the editors and conference program committee. Some conference presentations may not be available for publication. Additional papers and presentation recordings may be available online in the SPIE Digital Library at SPIEDigitallibrary.org.

The papers reflect the work and thoughts of the authors and are published herein as submitted. The publisher is not responsible for the validity of the information or for any outcomes resulting from reliance thereon.

Please use the following format to cite material from these Proceedings:

Author(s), "Title of Paper," in Advanced Sensor Systems and Applications VII, edited by Tiegen Liu, Shibin Jiang, Rene Landgraf, Proceedings of SPIE Vol. 10025 (SPIE, Bellingham, WA, 2016) Sevendigit Article CID Number.

ISSN: 0277-786X

ISSN: 1996-756X (electronic)

ISBN: 9781510604698

ISBN: 9781510604704 (electronic)

Published by

SPIE

P.O. Box 10, Bellingham, Washington 98227-0010 USA

Telephone +1 3606763290 (Pacific Time) · Fax +1 3606471445

SPIE.org

Copyright (C) 2016, Society of Photo-Optical Instrumentation Engineers.

Copying of material in this book for internal or personal use, or for the internal or personal use of specific clients, beyond the fair use provisions granted by the U.S. Copyright Law is authorized by SPIE subject to payment of copying fees. The Transactional Reporting Service base fee for this volume is $\$ 18.00$ per article (or portion thereof), which should be paid directly to the Copyright Clearance Center (CCC), 222 Rosewood Drive, Danvers, MA 01923. Payment may also be made electronically through CCC Online at copyright.com. Other copying for republication, resale, advertising or promotion, or any form of systematic or multiple reproduction of any material in this book is prohibited except with permission in writing from the publisher. The CCC fee code is 0277-786X/16/\$18.00.

Printed in the United States of America.

Publication of record for individual papers is online in the SPIE Digital Library.

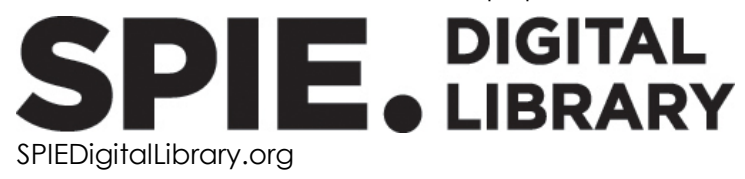

Paper Numbering: Proceedings of SPIE follow an e-First publication model. A unique citation identifier (CID) number is assigned to each article at the time of publication. Utilization of CIDs allows articles to be fully citable as soon as they are published online, and connects the same identifier to all online and print versions of the publication. SPIE uses a seven-digit CID article numbering system structured as follows:

- The first five digits correspond to the SPIE volume number.

- The last two digits indicate publication order within the volume using a Base 36 numbering system employing both numerals and letters. These two-number sets start with 00, 01, 02, 03, 04, 05, 06, 07, 08, 09, OA, OB ... 0Z, followed by 10-1Z, 20-2Z, etc. The CID Number appears on each page of the manuscript. 


\title{
Contents
}

\author{
vii Authors \\ ix Symposium Committees \\ xi Conference Committee
}

\section{SESSION 1 FIBER GRATING SENSORS}

1002502 Fiber Bragg grating-based temperature sensor for neutral gas in capacitively coupled plasmas (Invited Paper) [10025-1]

1002503 Simultaneous measurement of temperature and strain based on composite long-period fiber grating [10025-2]

1002504 Temperature insensitive measurements of displacement using fiber Bragg grating sensors [10025-3]

1002505 Sensing behavior of smart CFRP bar embedded with FBG and its application in stay cables [10025-4]

1002506 Optical fiber refractometer based on tapered tilted-fiber Bragg grating [10025-5]

\section{SESSION 2 OPTICAL SENSORS BASED ON MODE INTERFERENCE AND RESONATORS}

1002507 Interrelation of the effects caused by the rotation of the whispering gallery modes resonator (Invited Paper) [10025-6]

1002508 Temperature effect on refractive index sensing performance of a U-shape tapered plastic optical fiber [10025-7]

1002509 Thermal sensing performance of the nested fiber ring resonator [10025-8]

10025 OA The dispersion and spectrum output characteristics in series-coupled double-ring resonator [10025-10]

\section{SESSION 3 DISTRIBUTED AND REMOTE SENSORS}

10025 OB A gain compensation method for the long-range distributed fiber disturbance sensor (Invited Paper) [10025-11]

10025 OC Seismic wave detection system based on fully distributed acoustic sensing [10025-12]

10025 OD A study on frequency-shifted pulse light stability control for DP-MZM in coherent-OTDR [10025-13] 
10025 OE Positioning accuracy enhanced distributed disturbance fiber sensing system employing modified TDE algorithm [10025-14]

10025 OF Study on error budget of large deployable optical remote sensor [10025-15]

10025 OG Doppler laser radar for range and speed measurement of road targets [10025-16]

SESSION 4 METHODS FOR SENSOR INTERROGATION AND DATA PROCESSING

10025 0J Multi-sensor data fusion and estimation with poor information based on bootstrap-fuzzy model [10025-19]

10025 OK Tomographic reconstruction using 4 views and tunable diode laser [10025-20]

SESSION 5 CHEMICAL AND BIOLOGICAL SENSORS

$10025 \mathrm{OL}$ Experimental and modeling study of off-beam quartz-enhanced photoacoustic detection of nitrogen monoxide (NO) using a quantum cascade laser [10025-21]

10025 OM Amphiphilic block copolymer-based photonic platform towards efficient protein detection [10025-22]

10025 ON A photocoustic spectroscopy system for gas detection based on the multi-pass cell [10025-23]

1002500 Slotted-core photonic crystal fiber in gas-sensing application [10025-24]

10025 OQ Person identification by using 3D palmprint data [10025-26]

10025 OR Micro-capillary-based self-referencing surface plasmon resonance fiber-optic biosensor [10025-27]

10025 OS Application of LaserBreath-001 for breath acetone measurement in subjects with diabetes mellitus [10025-28]

SESSION 6 NEW MATERIALS, PRINCIPLES, METHODS, AND MODELING RELATED TO OPTICAL SENSING I

10025 OT Optical properties of $\mathrm{NaLuF}_{4}: \mathrm{Yb}^{3+}: \mathrm{Tm}^{3+} / \mathrm{Ho}^{3+}$ rare earth nanocrystals in microstructure hollow fiber [10025-29]

10025 OU Light propagation in strip and slot waveguide arrays for sensing [10025-30]

10025 OV Multimode interference structures as sensing elements integrated into Mach-Zehnder interferometers in polymer foils [10025-31]

10025 OW Optimizing the loss of one-dimensional photonic crystal towards high-sensitivity Blochsurface-wave sensors under intensity interrogation scheme [10025-32] 
10025 0X Rapid wasted-free microfluidic fabrication based on ink-jet approach for microfluidic sensing applications [10025-33]

10025 OY Magneto-optic sensor based on electrogyration compensation and single quartz crystal [10025-34]

SESSION 7 NEW MATERIALS, PRINCIPLES, METHODS, AND MODELING RELATED TO OPTICAL SENSING II

$100250 Z$ High sensitivity fiber-optic acoustic sensors (Invited Paper) [10025-35]

1002511 A novel quarter wave plate and its applications to the reflective fiber-optic current sensor [10025-37]

\section{POSTER SESSION}

1002512 Changes of shape of the whispering gallery modes resonators due to their movement in inertial space [10025-9]

1002514 The research of autonomous obstacle avoidance of mobile robot based on multi-sensor integration [10025-39]

1002516 Design of a micro uncooled infrared imaging system based on VOx IRFPA [10025-41]

1002517 Medical respiratory monitoring sensors based on microbend fiber loss [10025-42]

1002518 Development of fiber optical temperature sensor based on fluorescence lifetime [10025-43]

1002519 Research on high-temperature sensing characteristics based on modular interference of single-mode multimode single-mode fiber [10025-44]

10025 1A Measurement of optical fiber sensing parameter using FBG with WDM [10025-45]

10025 IC Modeling of a long-period fiber-optic grating assisted surface plasmon resonance refractive index sensor [10025-47]

10025 ID Porous silicon-based two-dimensional photonic crystal for biochemical sensing applications [10025-48]

10025 IF Demonstration of distributed fiber-optic temperature sensing with PM fiber using polarization crosstalk analysis technique [10025-50]

10025 IG Surface plasmon resonance sensor based on grapefruit-type photonic crystal fiber with silver nano-film [10025-51]

$10025 \mathrm{lH}$ Photonic crystal fiber sensor based on surface-enhanced Raman scattering for explosives detection [10025-52]

1002511 Torque transducer based on fiber Bragg grating [10025-53] 
10025 IJ A spherical-structure based fiber sensor for simultaneous measurement of ammonia gas concentration and temperature [10025-54]

$100251 \mathrm{~K}$ A refractive index sensor based on taper Michelson interferometer in multimode fiber [10025-55]

$10025 \mathrm{IL}$ Research into multispectral TDI-CCD imaging and fusion technology [10025-56]

10025 1M Detection of nitrogen dioxide by CW cavity-enhanced spectroscopy [10025-57]

10025 IN Remote canopy hemispherical image collection system [10025-58]

1002510 Concepts for compact mid-IR spectroscopy in photochemistry [10025-59] 


\section{Authors}

Numbers in the index correspond to the last two digits of the seven-digit citation identifier (CID) article numbering system used in Proceedings of SPIE. The first five digits reflect the volume number. Base 36 numbering is employed for the last two digits and indicates the order of articles within the volume. Numbers start with 00, 01, 02, 03, 04, 05, 06, 07, 08, 09, OA, OB...0Z, followed by 10-12, 20-2Z, etc.

\author{
Ahmed, K., 00 \\ Amarit, Rattasart, $0 \mathrm{X}$ \\ Asaduzzaman, S., 00 \\ Bai, Xuefei, $0 Q$ \\ Bao, Zhanjing, 03 \\ $\mathrm{Bi}$, Weihong, $1 \mathrm{~K}$ \\ Chen, Hongbing, $1 \mathrm{~L}$ \\ Chen, Rong, $1 \mathrm{H}$ \\ Chen, Shimeng, OR \\ Chen, Wenjie, OD \\ Chen, Xiaoli, OF \\ Chen, Xinglong, ON \\ Chen, XuDong, 03 \\ Chen, Zhuying, OS \\ Cui, He, OY \\ Cui, Jian, $1 \mathrm{~N}$ \\ Cui, Xiaojuan, ON \\ Cu-Nguyen, Phuong-Ha, 10 \\ Ding, Dongliang, IF \\ Ding, Zhenyang, OD \\ Dmitriyeva, Anna D., 07 \\ Dong, Fengzhong, 04, ON \\ Du, Kun, OW \\ Fang, Jianchao, OG \\ Farrell, Gerald, 1 J \\ Fei, Xianxiang, $1 \mathrm{C}$ \\ Feng, Shengwen, OC \\ Feng, Ting, $1 \mathrm{~F}$ \\ Filatov, Yuri V., 07, 12 \\ Fu, Guangwei, $1 \mathrm{~K}$ \\ Fu, Xinghu, $1 \mathrm{~K}$ \\ Gao, Dongyang, OA \\ Gao, Gang, 04 \\ Gao, Nan, OQ \\ Geng, Tao, 03 \\ Gibson, Thomas J., OM \\ Gleissner, Uwe, OV \\ Guo, Gaoran, 0C \\ Han, Baoling, 14 \\ Han, Daoman, 02 \\ Han, Fang, iN \\ Han, LUo, ON \\ Han, Wei, $1 \mathrm{~J}$ \\ Han, Ye-Xing, $1 M$ \\ $\mathrm{He}, \mathrm{Da}, \mathrm{lL}$ \\ He, Jiang, 03 \\ Hofmann, Meike, OV \\ Hu, Qihao, 03 \\ Hu, Shuyang, 11, 1A
}

\author{
Huang, Jianfen, OC \\ Huang, Jiang, $1 \mathrm{C}$ \\ Huang, Shujun, $\mathrm{OQ}$ \\ Jarujareet, Ungkarn, OX \\ Jia, Zhenhong, 1D \\ Jiang, Junfeng, O6, OB, OD, OE \\ Jiang, Shu, 11 \\ Jiang, Yue, OC \\ Jie, Guo, $1 M$ \\ Jin, Wa, $1 \mathrm{~K}$ \\ Jing, Ning, 08 \\ Kong, Weijing, OW \\ Kukaev, Alexander S., 12 \\ Langenecker, Alexa, OV \\ $\mathrm{Li}$, Changsheng, OY \\ Li, Fang, OC \\ Li, Feng, 05 \\ Li, Gui-lin, 18 \\ Li, Hanyang, OT \\ Li, Hui, 09, OA, OT \\ $\mathrm{Li}$, Jiang, $1 \mathrm{I}$ \\ $\mathrm{Li}$, Jingke, $1 \mathrm{H}$ \\ Li, Jun, 04 \\ Li, Lixia, OR \\ Li, Tao, 11 \\ Li, Xianjing, 17 \\ Li, Yan, OF \\ $L i$, Yingxin, OS \\ $\mathrm{Li}$, Zhe, ON \\ Li, Zhichen, OB \\ $\mathrm{Li}$, Zhihong, $1 \mathrm{~F}$ \\ Li, Zhili, OL \\ Li, Zixuan, 03 \\ Lian, Xiaokang, $1 \mathrm{~J}$ \\ Liang, Beiyan, 16 \\ Liao, Hao, $\mathrm{OZ}$ \\ Lin, Jiejun, 11 \\ Lin, Yanfang, OG \\ Liu, Bingyu, $1 \mathrm{~N}$ \\ Liu, Changlin, $1 \mathrm{~L}$ \\ Liu, Dejun, $1 \mathrm{~J}$ \\ Liu, Deming, $0 Z$ \\ Liu, Kun, 06, OB, OD, OE \\ Liv, Qiang, $1 \mathrm{~K}$ \\ Liu, Tiegen, 06, OB, OD, OE \\ Liv, Yongxin, 02 \\ Liu, Yun, OR \\ Liu, Zhishuang, OU \\ Liu, Zigeng, 02, OR
}


Lu, Ping, $O Z$

Lv, Xiaoyi, 1D

M., Osborn Oliver, OK

Ma, Chunyu, OB, OE

Ma, Qingyan, OU

$\mathrm{Ma}$, Zhe, OD

Mallik, Arun Kumar, $1 \mathrm{~J}$

Mao, Xuesong, OG

Miao, Tian, $\mathrm{OB}$

Mo, Jiaqing, 1D

Mueller, Claas, OV

Pan, Liang, OB, OE

Pang, Tao, ON

Paul, B. K., 00

Peng, Wei, 02, OR

Peng, Zhaozhuang, 19, 1A

Petropoulou, Afroditi, OM

Pispas, Stergios, OM

Qi, Fan, OU

Qi, Hongli, 11

Ren, Wei, OL

Riziotis, Christos, $\mathrm{OM}$

Semenova, Yuliya, $1 \mathrm{~J}$

Shalymov, Egor V., 07, 12

Shen, Feng, 05

Sherman, Stanislav, OV

Shi, Chao, OL

Song, Yong, 16

Song, Youchun, 16

Su, Hongxin, $1 \mathrm{~F}$

Sumriddetchkajorn, Sarun, OX

Sun, Weimin, 03

Sun, Lei, $1 \mathrm{~J}$

Sun, Meixiu, OS

Sun, Miao, 04

Sun, Naixun, OJ

Sun, Pengshuai, $0 \mathrm{~N}$

Sun, Xu, 05

T. M., Muruganandam, OK

Tang, Huai-Wu, IM

Tang, Yuquan, 04

Tao, Chuanyi, $1 \mathrm{H}$

Teng, Chuanxin, 08

Themistou, Efrosyni, OM

Tian, Xiuyun, 1C

Tong, Chengguo, 03

Venediktov, Vladimir Yu., 07, 12

Wan, Xuefen, IN

Wan, Yuhang, OW

Wang, Chuji, OS

Wang, Li, 19, 1 A

Wang, Shuang, OD, OW

Wang, Siwen, $1 \mathrm{~K}$

Wang, Tao, 06

Wang, Wenhua, 1C

Wang, Yanqing, $0 \mathrm{~J}$

Wang, Younian, 02

Wang, Yu, ON

Wang, Yuan, $1 G$

Wang, Yufei, OU
Wang, Yunxin, 11

Wang, Zhennan, OS

Wang, Ziyu, 10

Wei, Fangfang, $1 \mathrm{~J}$

Wu, Brian, ON

Wu, Qiang, 16

Wu, Qiang, $1 \mathrm{~J}$

Wu, Weina, $1 \mathrm{C}$

Wu, Yongfeng, 09, 0A, 0T

Xia, Hua, ON

Xiao, Yanfen, OV

Xie, Dingchao, 16

$\mathrm{Xu}$, Hongbin, 05

$X u$, Shengming, 04

$\mathrm{Xu}$, Tuanwei, OC

Xue, Meng, 06

Yan, Huanhuan, 19, 1A

Yang, Jian, 17

Yang, Shuang, 04

Yang, Wenlei, 03

Yang, Yang, $\mathrm{OC}$

Yang, $Y i, 1 N$

Yang, Yue, 16

Yao, Jianquan, $1 G$

Yao, X. Steve, $1 \mathrm{~F}$

Yu, Changqiu, 09, 0A, 0T

Yu, Fangda, 08

Yu, Zhe, 06

Yu, Zhi-Wei, $1 \mathrm{M}$

Yuan, Ping, 09, 0A, OT

Yue, Ming-yang, 18

Zappe, Hans, OV, 10

Zhang, Jiangpeng, $1 \mathrm{~K}$

Zhang, Lei, 1G

Zhang, Ruibin, 11

Zhang, Tao, OG

Zhang, Tuo, 09, 0T

Zhang, Xiaoqing, $0 \mathrm{~J}$

Zhang, Xinpu, 02

Zhang, Xuan, OY

Zhang, Xuezhi, OD

Zhang, Yundong, 09, 0A, 0T

Zhang, Zhirong, ON

Zhang, Zonghua, $\mathrm{OQ}$

Zhao, Ming, 14

Zhao, Weigang, 05

Zhao, Wenhui, OW

Zhao, Xiaomeng, OS

Zhao, Yong, 17, 18

Zhao, Ziwei, IF

Zheng, Hong-kun, 18

Zheng, Jie, 08

Zheng, Wanhua, OU

Zheng, Yibo, $1 \mathrm{G}$

Zheng, Zheng, oW

Zhou, Jianyong, $1 \mathrm{~L}$

Zhouxiao, Liuting, 17 


\title{
Symposium Committees
}

\author{
General Chairs \\ Robert Lieberman, SPIE President, Lumoptix, LLC (United States) \\ Guangcan Guo, Chinese Optical Society President, University of \\ Science and Technology of China (China) \\ General Co-chairs \\ Arthur Chiou, National Yang-Ming University (Taiwan, China) \\ Jianlin Cao, China Ministry of Science and Technology (China) \\ Junhao Chu, Shanghai Institute of Technical Physics (China) \\ Technical Program Chairs \\ Songlin Zhuang, University of Shanghai for Science and Technology \\ (China) \\ Xingde Li, Johns Hopkins University (United States) \\ Technical Program Co-chairs \\ Bingkun Zhou, Tsinghua University (China) \\ Qiming Wang, Institute of Semiconductors (China) \\ Tianchu Li, National Institute of Metrology (China) \\ Wei Huang, Nanjing University of Technology (China) \\ Ying Gu, PLA General Hospital (China) \\ Huilin Jiang, Changchun University of Science and Technology \\ (China) \\ Local Organizing Committee Chair \\ Qihuang Gong, Peking University (China)
}


Local Organizing Committee Co-chairs

Xu Liu, Zhejiang University (China)

Daoyin Yu, Tianjin University (China)

Guoqiang Ni, Beijing Institute of Technology (China)

Shusen Xie, Fujian Normal University (China)

Xiaomin Ren, Beijing University of Posts and Telecommunications

(China)

General Secretary

Yan Li, Chinese Optical Society/Peking University (China)

Local Organizing Committee

Zhiping Zhou, Peking University (China)

Changhe Zhou, Shanghai Institute of Optics and Fine Mechanics, CAS

(China)

Qingming Luo, Huazhong University of Science and Technology

(China)

Chongxiu Yu, Beijing University of Posts and Telecommunication

(China)

Hongda Chen, Institute of Semiconductors (China)

Yongtian Wang, Beijing Institute of Technology (China)

Yiping Cui, Southeast University (China)

Xuping Zhang, Nanjing University (China)

Feijun Song, Daheng Corporation (China)

Cunlin Zhang, Capital Normal University (China)

Yanting Lu, Nanjing University (China)

Yuejin Zhao, Beijing Institute of Technology (China)

Chunqing Gao, Beijing Institute of Technology (China)

Tiegen Liu, Tianjin University (China)

Xiaocong Yuan, Nankai University (China)

Weimin Chen, Chongqing University (China)

Zhongwei Fan, Academy of Optoelectronics (China)

Hanyi Zhang, Tsinghua University (China)

Lan Wu, Zhejiang University (China)

Yongsheng Zhang, University of Science and Technology of China (China)

Hong Yang, Peking University (China)

Xiaoying Li, Tianjin University (China)

Wei Xiong, Chinese Optical Society (China) 


\title{
Conference Committee
}

\author{
Conference Chairs
}

Tiegen Liu, Tianjin University (China)

Shibin Jiang, AdValue Photonics, Inc. (United States)

Rene Landgraf, Fraunhofer-Institut für Photonische Mikrosysteme

(Germany)

Conference Program Committee

Xiaoyi Bao, University of Ottawa (Canada)

Rongshen Chen, The University of Birmingham (United Kingdom)

Kin-Seng Chiang, City University of Hong Kong (Hong Kong, China)

Brian Culshaw, University of Strathclyde (United Kingdom)

Xudong Fan, University of Michigan (United States)

Claire Gu, University of California, Santa Cruz (United States)

Baiou Guan, Jinan University (China)

Zuyuan He, Shanghai Jiao Tong University (China)

Wei Jin, The Hong Kong Polytechnic University Shenzhen Research Institute (China)

Deming Liu, Huazhong University of Science and Technology (China)

Niels Neumann, Technische Universität Dresden (Germany)

Li Pei, Beijing Jiaotong University (China)

Gang-Ding Peng, The University of New South Wales (Australia)

Xueguang Qiao, Northwest University (China)

Yunjiang Rao, University of Electronic Science and Technology of China (China)

Tobias Schuster, Technische Universität Dresden (Germany)

Anbo Wang, Virginia Polytechnic Institute and State University (United States)

Tingyun Wang, Shanghai University (China)

Hai Xiao, Clemson University (United States)

Steve Yao, General Photonics Corporation (United States)

Paul Kit-Lai Yu, University of California, San Diego (United States)

Li-Bo Yuan, Harbin Engineering University (China)

Session Chairs

1 Fiber Grating Sensors

Vladimir Y. Venediktov, Saint Petersburg Electrotechnical University "LETI" (Russian Federation) 
2 Optical Sensors Based on Mode Interference and Resonators

Tao Weng, Tianjin University (China)

3 Distributed and Remote Sensors

Tiegen Liu, Tianjin University (China)

4 Methods for Sensor Interrogation and Data Processing

Kun Liu, Tianjin University (China)

5 Chemical and Biological Sensors

Rene Landgraf, Fraunhofer-Institut für Photonische Mikrosysteme (Germany)

6 New Materials, Principles, Methods, and Modeling Related to Optical Sensing I

Ping Lu, Huazhong University of Science and Technology (China)

7 New Materials, Principles, Methods, and Modeling Related to Optical Sensing II

Ping Lu, Huazhong University of Science and Technology (China) 